\title{
Adaptation to exercise in the rat: Lack of associative control
}

\author{
MITCHELL M. METZGER and DAVID C. RICCIO \\ Kent State University, Kent, Ohio
}

\begin{abstract}
A procedure in which female Long-Evans rats were swum to a criterion of fatigue was utilized to assess the contextual determinants of adaptation to exercise. In Experiment 1, it was observed that rats given four exercise exposures demonstrated significant adaptation to the treatment, as indicated by reliably longer swimming times over the course of exercise exposure. Furthermore, the change in swimming times reflected in these subjects was not due to maturation or growth over the course of the experiment, as their body weights before and after the experiment did not reliably differ. Experiment 2 addressed whether adaptation to swimming exercise was susceptible to a context shift effect, as is tolerance to drugs and adaptation to other homeostatic disturbances (e.g., hypothermia). Using a withinsubject design, Experiment 2 demonstrated that a shift in contextual stimuli (a procedure that reliably disrupts drug tolerance and hypothermia adaptation) failed to disrupt established adjustment to exercise. The results of these experiments suggest that adaptation to exercise may be mediated by different mechanisms than adaptation to other disturbances (e.g., drugs, hypothermia), since an alteration in contextual stimuli did not appear to be detrimental to established exercise adaptation with this paradigm.
\end{abstract}

In the traditional view of drug tolerance, it is hypothesized that tolerance to drugs is mediated purely by pharmacological and/or metabolic processes. However, in recent years, a voluminous body of literature has implicated associative processes in the development of drug tolerance as well (Siegel, 1975, 1976, 1989). In a seminal study, Siegel (1975) demonstrated that tolerance to morphine could be disrupted simply by administering the drug in a context not previously paired with morphine administration. As a result of this and other findings, Siegel proposed that Pavlovian associative processes occurred during temporal and spatial pairing of morphine and the physical environment, and that these learned associations could account for the situational specificity of manifested tolerance. This account of tolerance clearly conflicts with the traditional view of drug tolerance, in which it is hypothesized that a shift in ambient stimuli should have little or no effect on the manifestation of tolerance at the time of test.

In Siegel's Pavlovian conditioning model, the contextual cues present at the time of drug administration act as the conditioned stimulus (CS), and the systemic effects of the drug serve as the unconditioned stimulus (US). Thus, tolerance is a direct product of context (CS) and systemic drug effect (US) pairings. In order for tolerance to develop, therefore, the subject must have a consistent set of (con-

The authors wish to thank R. Toulon and D. Spray for helpful assistance with data collection. The research reported here was supported in part by National Institute of Mental Health Grant MH37535 to D.C.R. Care of animals was approved by Kent State University's Animal Care and Use Committee. Correspondence should be addressed to M. M. Metzger, Department of Psychology, Kent State University, Kent, OH 44242-0001 (e-mail: mmetzger@kentvm.kent.edu). textual) cues that reliably predict administration of the drug. Importantly, according to this Pavlovian model, presentations of the CS elicit compensatory conditioned responses (CCR) that grow in strength with repeated CS-US pairings. The CCR is an action opposite the systemic effect of the drug, and tolerance develops because the CCR opposes the US drug effect. Hence, when the CS is absent (different context), the CCR fails to be elicited, and what had previously appeared as tolerance does not occur (i.e., a context shift effect).

In addition, some evidence suggests that Pavlovian associative processes influence not only tolerance to some drugs but also adaptation to other homeostatic disruptions. For example, Riccio, MacArdy, and Kissinger (1991) studied the adaptation of rats to repeated exposure to cold temperatures and observed that adaptation to the cold (as reflected in a less severe hypothermia over repeated exposures) was disrupted when animals experienced the same cold temperatures in the presence of novel contextual stimuli. This influence of environmental context was further investigated by Kissinger and Riccio (1995), who reported that altering the context during each day's exposure to cold temperature slowed adaptation to that temperature. Moreover, Goodison and Siegel (1995) have reported that recovery of suppressed feeding produced by injections of cholecystokinin (CCK) was influenced by the presence of environmental stimuli associated with $\mathrm{CCK}$ administration. When CCK was injected immediately prior to feeding, tolerance to the suppressive effect of CCK was observed, whereas no tolerance to CCK was observed if there was a noncontingent relationship between feeding and drug administration. These results suggest that tolerance to morphine and other drugs, as well as adaptation to cold, can be influenced by associative learning processes. 
There is considerable evidence that organisms can adapt to a wide variety of environmental challenges, ranging from adjustment to cold and heat disturbances to physiological adaptation to changes in altitude (Samueloff \& Yousef, 1987; Yousef, Horvath, \& Bullard, 1972). The purpose of the present research was to examine adaptation to a different type of environmental challenge, forced exercise, and to determine the influence of associative processes on such a disturbance. Previous experiments on adaptation to exercise have measured physiological responses such as heart rate (Tipton, Barnard, \& Tcheng, 1969) and changes in plasma lipoproteins (Papadopoulos, Bloor, \& Standefer, 1969), but have not examined behavioral measures of adaptation (such as the amount of time swimming). In Experiment 1, female rats were studied in the same environment for 4 days during which they were required to swim to a criterion of fatigue; increased swim times over the four exposures were consistent with the notion of an adaptation process to forced exercise. In Experiment 2, female rats were again forced to swim until fatigued in the same environment for either 4 or 5 days, and were then tested on an additional day in a novel environment. Results from Experiment 2 showed that adaptation to forced swimming was similar in both environments, suggesting that associative processes did not mediate adaptation to forced swimming.

\section{EXPERIMENT 1}

This experimient addressed whether rats can develop adaptation to a forced swimming procedure as they adapt to drugs and hypothermia. In contrast with previous experiments, a behavioral technique for examining adaptation to forced exercise was employed in the present research so that direct measurement of functional adaptive processes could be observed rather than indirect variables of adaptation, such as metabolic and physiological changes (cf. Papadopoulos et al., 1969; Tipton et al., 1969). If subjects adjusted to the exercise, an increase in the time to reach the fatigue criterion should be seen with repeated exposures.

\section{Method}

\section{Subjects}

Sixteen female Long-Evans rats obtained from the Kent State University breeding colony served as subjects, and they were between 210 and $270 \mathrm{~g}$ at the beginning of the experiment. All subjects were housed individually in hanging wire mesh cages in a colony room maintained on a 15:9-h light:dark cycle and a temperature of approximately $70^{\circ} \mathrm{F}$. Food and water were available on demand, and all experimentation took place during the middle half of the light cycle. All subjects had previously received passive avoidance training unrelated to the present experiment.

\footnotetext{
Apparatus

The swim tank consisted of a rectangular Rubbermaid trash can with approximate inside dimensions of $30 \times 30 \times 58 \mathrm{~cm}$, and the tank was filled with room-temperature water up to approximately $10 \mathrm{~cm}$ from the top. A 9-g fishing weight was fastened to the base of each subject's tail to keep subjects swimming rather than simply floating.
}

Standard stopwatches were used to record total amount of time swimming in the tank and number of consecutive seconds underwater.

\section{Procedure}

The context in which all experimental manipulations took place was a room of approximately $2.4 \times 1.6 \times 2.7 \mathrm{~m}$ located inside the colony room. The walls of the room were painted yellow, and it was illuminated by four fluorescent tubes located on the ceiling.

Subjects were handled for 2-3 min, weighed, and divided into two groups 1 day prior to the first swimming session. During the adaptation phase of the experiment, each rat was individually brought into the experimental room in a standard plastic shoebox and the 9-g fishing weight was attached to the base of the rat's tail. Subjects were then placed in the swim tank and swum to the point of moderate fatigue (as measured by 15 consecutive seconds without surfacing). After the subjects reached criterion, they were immediately removed from the swim tank, towel dried, returned to their home cages, and the amount of time spent swimming in the tank was recorded.

The swim group received four forced swimming sessions that were administered on a Monday-Wednesday-Friday schedule (the first session occurred on a Wednesday). The control group received only one session, which was administered on the 4th day of swimming exposure for the swim group.

\section{Results and Discussion}

The mean amount of time swimming for the swim and control groups is presented in Figure 1. As can be seen from the figure, the swim group that was given four exposures to exercise demonstrated adaptation to the treatment, as reflected in longer swimming times as the days progressed. This impression was confirmed by an analysis of variance (ANOVA), which revealed that the swim group spent a significantly longer time swimming in the tank for each successive session $[F(3,21)=8.78, p<.01]$. Furthermore, the swimming time of the control group (which was given only one exercise exposure) was reliably different from that of the swim group on Day $4(T=-3.13, p<.01)$, suggesting that the increased swimming time of the swim group was a result of adaptation to the treatment rather than weight gain or growth. Moreover, a within-group $T$ test computed on body weights of the subjects before and after exposure to swimming clearly argued against a growth interpretation, since there were no reliable differences between the two measures $(T=-1.17, p>.10)$. Additionally, the mean swimming time of the control group did not statistically differ from that of the swim group on Day 1 of the experiment $(T=-.54, p>.10)$.

The results of Experiment 1 indicate that rats were able to readily adapt to a forced exercise exposure, as reflected behaviorally in an increase in swimming time over the course of repeated exposure to the swimming treatment. Whether this adaptation was the result of muscle strengthening and other physiological changes or involved the types of learning processes seen in drug tolerance and thermoregulatory adaptation is addressed in Experiment 2.

\section{EXPERIMENT 2}

One manipulation that has proven useful in detecting associative processes in adaptation to homeostatic disturbances has been to test adapted subjects in a different 


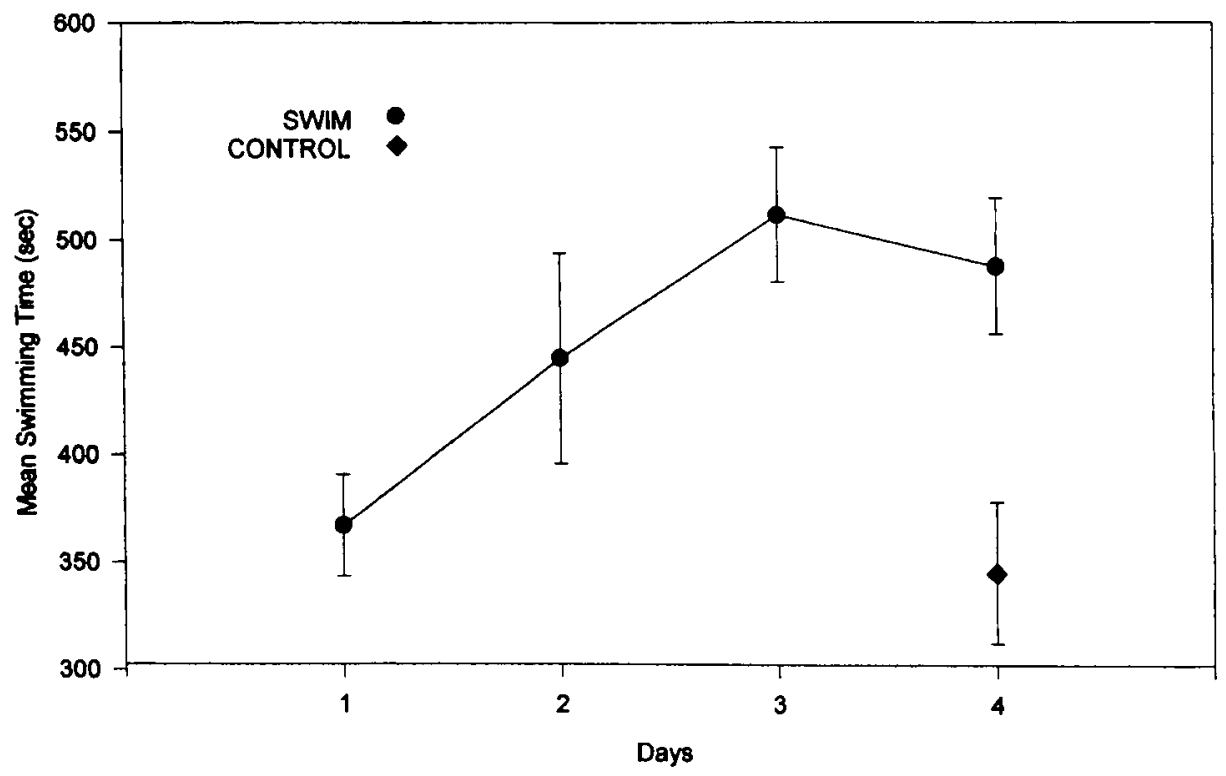

Figure 1. Mean swimming time over the adaptation phase for the swim group and mean swimming times of the control group on Day 4 of Experiment 1. (Error bars = standard error of the mean.)

(shifted) context. As noted in the introduction, altering the environmental context has been demonstrated to disrupt both drug tolerance (see, e.g., Siegel, 1975; see also Poulos \& Cappell, 1991, for a recent review) and thermoregulatory adaptation (Kissinger \& Riccio, 1995; Riccio et al., 1991). Accordingly, Experiment 2 was designed to determine if a shift in ambient stimuli would disrupt performance in subjects adapted to the swimming exposures.

\section{Method \\ Subjects \\ Sixteen female Long-Evans rats from the Kent State University breeding colony that were between 245 and $300 \mathrm{~g}$ at the beginning of the experiment served as subjects. They were housed and main- tained in a manner similar to that described in Experiment 1. Food and water were available on demand, and all experimental manipu- lation took place during the middle half of the light cycle. All sub- jects had previously received passive avoidance training and condi- tioned taste aversion conditioning, treatments unrelated to the present experiment.}

\section{Apparatus \\ The same swim tank that was used in Experiment 1 was used in the present experiment. However, since the rats in Experiment 2 weighed slightly more than the subjects in Experiment 1, an 11-g fishing weight was fastened to each subject's tail (as opposed to the weight used in Experiment 1). Standard stopwatches were used to record total amount of time spent swimming to the criterion of fatigue.}

\section{Procedure}

The swimming adaptation context was identical to that used in Experiment 1. However, the room used for the context shift manipulation differed from the adaptation context in size, color, illumination, odor, and noise level. The shift room $(3.3 \times 1.8 \times 2.7 \mathrm{~m})$ was slightly larger than the adaptation room and was located in the animal lab. The walls were painted orange and it was illuminated by a $25-\mathrm{W}$ red light bulb suspended $30 \mathrm{~cm}$ above the water surface of the swim tank. Furthermore, a lemon odor and white noise were present in the shifted context. This manipulation of changing the contextual attributes of the experimental room has been reported to be disruptive in both drug tolerance (Siegel, 1989) and hypothermia adaptation experiments (Kissinger \& Riccio, 1995; Riccio et al., 1991).

As in Experiment 1, all subjects were handled for $2-3 \mathrm{~min}$, weighed, and divided into two groups 1 day prior to experimental manipulation. Rats in the Shift 5 group received 4 days of swimming exposure and subjects in the Shift 6 group received 5 days of swimming exposure, as in Experiment 1. (All sessions during this phase of the experiment occurred in the swimming adaptation context.) As in Experiment 1 , sessions were conducted on a Monday-WednesdayFriday schedule with the experiment starting on a Wednesday.

On Day 5 of the experiment, animals in the Shift 6 group received their swimming exposure in the swimming adaptation context, while rats in the Shift 5 group received their exercise exposure in the shifted context. Rats in the Shift 5 group were taken individually on a cart to the shifted context and were administered the exercise exposure in the presence of altered contextual stimuli. (The only difference during this exposure to the swimming exercise was the presence of shifted ambient stimuli.) On Day 6 of the experiment, the Shift 5 group was returned to the swimming adaptation context for exercise exposure, while the Shift 6 group was administered swimming exposure in the shifted context (as described above). This procedure of administering the shift condition to only one group of subjects per day was utilized so that both a within- and between-groups measure could be used in determining if the shift in contextual stimuli was detrimental to established adaptation.

\section{Results and Discussion}

Figure 2 presents the mean swimming time for the Shift 5 and Shift 6 groups across Days $1-6$ of the experiment. As in Experiment 1, scores for each group were plotted as the mean swimming time. Both the Shift 5 and Shift 6 groups demonstrated adaptation to exercise as reflected in longer swimming times with repeated exposure to the treatment, an outcome replicating that of Experiment 1. The Shift 5 group demonstrated reliably longer latencies to reach the fatigue criterion over 4 days of swimming ex- 


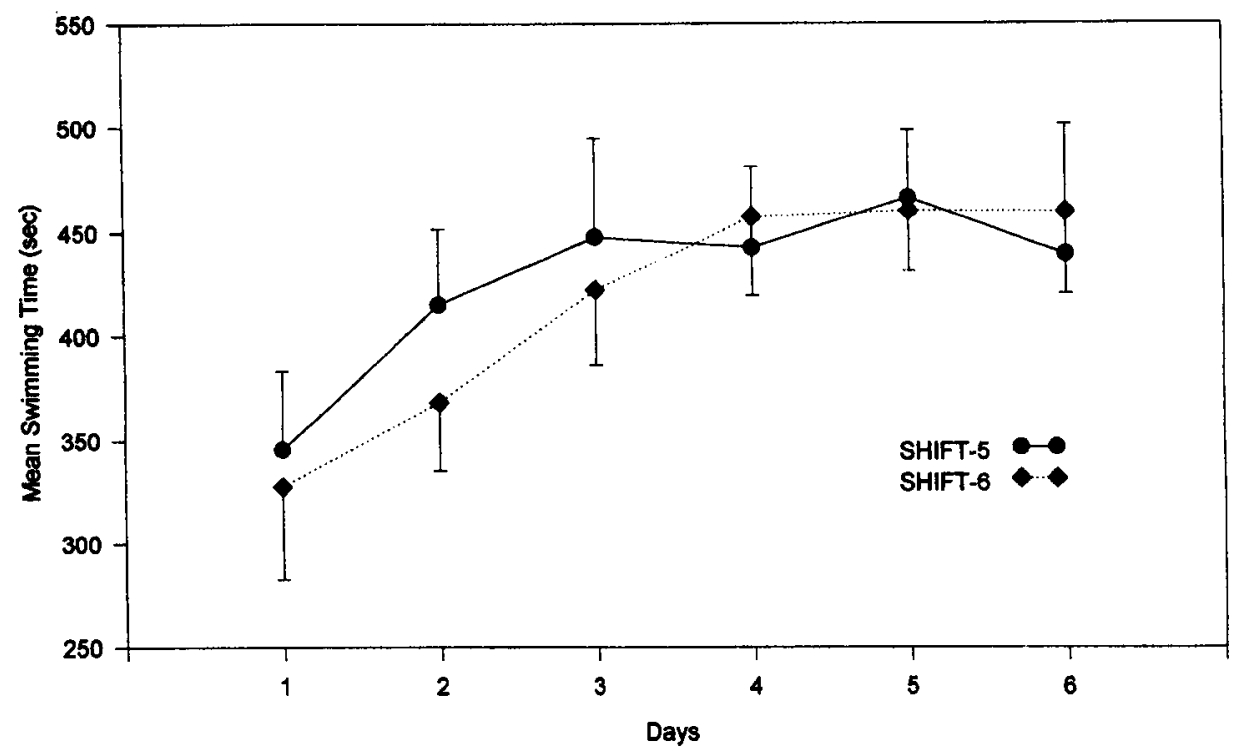

Figure 2. Mean swimming time for the Shift 5 and Shift 6 groups over the adaptation phase and the test in the shifted context. (Error bars $=$ standard error of the mean.)

posure $[F(3,21)=9.15, p<.01]$, while the Shift 6 group showed comparable adaptation to the swim stress over 5 days $[F(4,28)=9.48, p<.01]$. Furthermore, the rate at which subjects in the Shift 5 and Shift 6 groups acquired adaptation did not statistically differ over the first four exercise exposures, as confirmed by a group $\times$ days ANOVA $[F(3,42)=.94, p>.10]$.

The results of the tests in the shifted context indicated that adaptation to this type of disturbance was not adversely effected by a change in ambient stimuli. For the Shift 5 group, a shift in contextual stimuli on Day 5 did not reduce established adaptation to exercise, since swimming times on Day 5 were not reliably lower than those on Day 4 ( $T=$ $-1.19, p>.10$ ). Furthermore, swimming times on the shift day were still reliably higher than swimming times on Day 1 of the experiment for the Shift 5 group $(T=-4.78$, $p<.01)$. A similar pattern of results was obtained when scores for the Shift 6 group were examined. Swimming times in the shifted context for the Shift 6 group were not significantly lower than the scores from the 5 th day of the experiment $(T=.02, p>.10)$. Moreover, swimming times on the shift day were still significantly higher than the swimming times on the 1st day of swimming exposure for the Shift 6 group $(T=-3.13, p<.05)$.

Between-groups comparisons also failed to detect any differences between subjects tested in the shifted context and those tested in the swimming adaptation context. A comparison between the groups on Day 5 of the experiment, in which the Shift 5 group was administered exercise exposure in the shifted context and group Shift 6 received swimming exposure in the adaptation context, failed to reveal any reliable differences between the groups $(T=.15, p>.10)$. Likewise, an examination of the swimming times on Day 6 of the experiment (in which the Shift 6 group was tested in the shifted context and the Shift 5 group was tested in the adaptation context) failed to confirm the presence of a context shift effect $(T=.28, p>.10)$.

As in Experiment 1, developmental growth or maturation did not appear to play a role in the acquisition of adaptation to swimming exposure in Experiment 2. Before the start of the experiment, the mean weight of the subjects was $272.5 \mathrm{~g}$, and their mean weight on the last day of the experiment was $274.3 \mathrm{~g}$. Clearly, this trivial increase in subject body weight did not mediate adaptation to exercise in this experiment.

\section{GENERAL DISCUSSION}

This research was designed to examine the contextual determinants of adaptation to repeated exercise exposures in rats. Experiment 1 demonstrated that rats can quickly adjust to exercise, as indicated by a significant increase in swimming times over the course of exercise administration. This finding is consistent with previous reports of tolerance in rats with repeated exposures to drugs (Poulos \& Cappell, 1991; Siegel, 1989) or adaptation to hypothermia treatment (Riccio et al., 1991). The results of Experiment 2 showed that if adapted rats were administered exposure to exercise in a novel context, there was no disruptive effect on established adaptation to the swimming treatment. This finding is inconsistent with previous reports on drug tolerance and thermoregulatory adaptation. In earlier studies, it has been reported that a change in ambient contextual stimuli disrupts both drug tolerance (Siegel, 1989) and adaptation to hypothermia (Kissinger \& Riccio, 1995; Riccio et al., 1991).

The effects of intense exercise on organisms have been studied previously, but these experiments typically measured biological and/or metabolic functions rather than behavioral indices of adaptation. Tipton et al. (1969) re- 
ported that when hypophysectomized rats were administered treadmill running exercise, their resting heart rates after $30-40$ days of training were significantly lower than those of nontrained control rats. Papadopoulos et al. (1969) found similar changes in body lipid concentrations as a result of intense exercise training. They administered swimming exercise to rat subjects for $4 \mathrm{~h}$ daily for a 28 -day period and reported that changes in plasma lipids and lipoproteins occurred as a result of this type of strenuous exercise. The present experiments differed from these earlier experiments since we examined a behavioral index of adaptation (swimming time) rather than a biological or metabolic process. The results, however, were similar in that our index of adjustment to the exercise exposures also changed over the course of repeated administrations of the exercise treatment. Of particular note is the rapidity with which adaptation developed to the relatively brief (roughly 5-7 $\mathrm{min}$ ) homeostatic challenges in the present experiments.

The finding that a shift in ambient stimuli had no detrimental effect on established swimming adaptation suggests that the adjustment revealed in our subjects was controlled by nonassociative processes. If adaptation to the swimming challenge was mediated by associative learning mechanisms, a change in contextual stimuli should have been disruptive to established adaptation. One plausible alternative explanation of the negative results in Experiment 2 is that the contextual stimuli that were shifted were not sufficiently salient, and if the "proper" cues had been altered, a disruption in established adaptation would have been observed. However, the contextual cues that were altered in Experiment 2 (room size, illumination, odor, and noise level) have been demonstrated to be effective in our lab in disrupting tolerance to morphine (Feinberg \& Riccio, 1990 ) and adaptation to hypothermia treatment (Kissinger \& Riccio, 1995; Metzger, Kissinger, Harrod, Riccio, \& Toulon, 1995; Riccio et al., 1991). It is not clear why the alteration of these same cues would be effective in disrupting adaptation to other types of homeostatic disturbances but not to forced exercise, as in the present case. The possibility remains, however, that a larger change in environmental context than what was used in the present experiments would be required to disrupt adaptation to exercise with this paradigm.

Another plausible explanation for the negative results of Experiment 2 is that the stressfulness of the swimming exposure may have overshadowed the contextual cues present during the adaptation (and shift) phase of the experiment. In one experiment, Walter and Riccio (1983) reported that tolerance to the analgesic effects of morphine was susceptible to overshadowing when two external cues differed in intensity of salience. Furthermore, such overshadowing can also be achieved by internal stimuli, as demonstrated by Greeley, Le, Poulos, and Cappell (1984) in a study of adaptation to the effects of alcohol administration. In their study, one group of rats received a low dose of alcohol followed $60 \mathrm{~min}$ later by a high dose of alcohol during tolerance acquisition, while another group of rats received the low and high doses of alcohol in an unpaired manner. When tested for adaptation to the hypothermic ef- fect of the high dose of alcohol, only the group that had received the two doses in a paired manner demonstrated reliable adaptation. Importantly, adaptation was observed in the paired group only if the low dose of alcohol preceded the high dose during the test for tolerance. This result suggests that the cues produced by the low dose of alcohol overshadowed any external contextual cues that may have predicted administration of the drug. The results of Walter and Riccio (1983) and Greeley et al. (1984) suggest that overshadowing may occur in studies of drug tolerance; however, whether or not an overshadowing account is applicable to the present data remains undetermined.

Previous research has demonstrated that rats can sustain very long periods of forced swimming (Richter, 1957), and the 15 -sec criterion that we employed in this study was chosen with this finding in mind. The use of a forced swimming procedure as an environmental challenge has been widely used in a number of different paradigms and in somewhat different ways (see Mabry, Gold, \& McCarty, 1995; Papadopoulos et al. 1969; Richter, 1957; Tan, Morimoto, Sugiura, Morimoto, \& Murakami, 1992). As noted, we used a variation of the forced swimming procedure in the present research so that a behavioral index of adaptation could be measured, rather than using a physiological and/or metabolic index of adaptation (cf. Papadopoulos et al., 1969; Tipton et al., 1969).

Finally, the results of Experiment 2 are similar to those of an earlier study by Kokkinidis (1986) in which it was reported that a shift in contextual stimuli was not disruptive to established cold adaptation in mice. However, Kokkinidis employed a bout of swimming in cold water to induce adjustment to hypothermia. Thus, both exercise adaptation and cold adaptation may have contributed to the reduced severity of hypothermia observed over the 15 days of daily exposure. Accordingly, the lack of a context shift effect in Kokkinidis's study may have represented the nonassociative aspects of exercise adaptation, as in the present research.

In summary, it appears that rats can adapt to repeated exercise exposures as well as they can adapt to repeated drug administrations or homeostatic disruptions. However, the lack of contextual control over exercise adaptation in the present paradigm suggests that this outcome is mediated by mechanisms other than associative learning processes.

\section{REFERENCES}

Feinberg, G., \& Riccio, D. C. (1990). Changes in memory for stimulus attributes: Implications for tests of morphine tolerance. Psychological Science, 1, 265-267.

Goodison, T., \& SiEGEL, S. (1995). Learning and tolerance to the intake suppressive effect of cholecystokinin in rats. Behavioral Neuroscience, 109, 62-70.

Greeley, J., Le, D. A., Poulos, C. X., \& Cappell, H. (1984). Alcohol is an effective cue in the conditional control of tolerance to alcohol. Psychopharmacology, 83, 159-162.

KIsSingeR, S. C., \& Riccio, D. C. (1995). Stimulus conditions influencing the development of tolerance to repeated cold exposure in rats. Animal Learning \& Behavior, 23, 9-16.

KoKKINIDIS, L. (1986). Adaptation to cold swim stress-induced hypothermia: Absence of Pavlovian conditional tolerance. Behavioral Processes, 12, 57-66. 
MABRY, T. R., GolD, P. E., \& MCCARTY, R. (1995). Age-related changes in plasma catecholamine responses to acute swim stress. Neurobiology of Learning \& Memory, 63, 260-268.

Metzger, M. M., Kissinger, S. C., Harrod, S. B., Riccio, D. C., \& Toulon, R. (1995). Failure to extinguish associative control of cold tolerance in rats. Unpublished manuscript.

Papadopoulos, N. M., Bloor, C. M., \& Standefer, J. C. (1969). Effects of exercise and training on plasma lipids and lipoproteins in the rat. Journal of Applied Physiology, 26, 760-763.

Poulos, C. X., \& CAPpell, H. (1991). Homeostatic theory of drug tolerance: A general model of physiological adaptation. Psychological Review, 98, 390-408.

Riccio, D. C., MacArdy, E. A., \& Kissinger, S. C. (1991). Associative processes in adaptation to repeated cold exposures in rats. Behavioral Neuroscience, 105, 599-602.

RICHTER, C. P. (1957). On the phenomenon of sudden death in animals and man. Psychosomatic Medicine, 19, 191-198.

SAMUELoff, S., \& Yousef, M. K. (EDS.) (1987). Adaptive physiology to stressful environments. Boca Raton, FL: CRC Press.

SIEGEL, S. (1975). Evidence from the rat that morphine tolerance is a learned response. Journal of Comparative \& Physiological Psychology, 89, 498-506.
SIEGEL, S. (1976). Morphine analgesic tolerance: Its situation specificity supports a Pavlovian conditioning model. Science, 193, 323-325.

SiEgEL, S. (1989). Pharmacological conditioning and drug effects. In A. J. Goudie \& M. Emmett-Ogelsby (Eds.), Psychoactive drugs (pp. 115-180). Clifton, NJ: Humana Press.

Tan, N., Morimoto, K., Sugiura, T., Morimoto, A., \& Murakami, N. (1992). Effects of runining training on the blood glucose and lactate in rats during rest and swimming. Physiology \& Behavior, 51, 927-931.

Tipton, C. M., Barnard, J., \& Tcheng, T. (1969). Resting heart rate investigations with trained and nontrained hypophysectomized rats. Journal of Applied Physiology, 26, 585-588.

WALTER, T. A., \& Riccio, D. C. (1983). Overshadowing effects in the stimulus control of morphine analgesic tolerance. Behavioral Neuroscience, 97, 658-662.

Yousef, M. K., Horvath, S. M., \& Bullard, R. W. (Eds.) (1972). Physiological adaptations: Desert and mountain. San Diego: Academic Press.

(Manuscript received April 21, 1995; revision accepted for publication October 25,1995 .) 